\title{
HIGH VELOCITY FLYER PLATE LAUNCH CAPABILITY ON THE SANDIA Z ACCELERATOR
}

\author{
C.A. HALL*, M.D. KNUDSON*, J.R. ASAY*, R. LEMKE*, AND B. OLIVER** \\ 'Sandia National Laboratories, Shock Physics Applications Department., Albuquerque, NM, 87185-1181, USA, \\ **Mission Research Corporation, Albuquerque, NM 87110, USA
}

\begin{abstract}
A method has been developed for launching plates useful for equation of state (EOS) studies to high velocities using fast pulsed power on the Sandia National Laboratories $Z$ Accelerator. The technique employs magnetic pressure developed in an insulating gap between the anode and cathode of the machine to provide smoothly increasing, quasi-isentropic loading to plates of $9-12 \mathrm{~mm}$ in diameter and hundreds of microns thickness. Successful launches of titanium to $\sim 12 \mathrm{~km} / \mathrm{s}$, aluminum to $\sim 13 \mathrm{~km} / \mathrm{s}$, and copper to $\sim 10 \mathrm{~km} / \mathrm{s}$ have been demonstrated. The plates were monitored through the entire launch process with both conventional and spatially resolved velocity interferometry to obtain acceleration histories and impact profiles. Impacts of the flyers into aluminum wedges were also performed to experimentally determine final plate thickness and to obtain some estimates of integrity upon impact. Initial indications are that the plates are intact, slightly bowed, and at essentially ambient state.
\end{abstract}

Keywords: place keywords here, indented same as the Abstract, use $10 \mathrm{pt}$ font, keywords are separated by commas, single space between the Abstract and double space to the Introduction.

\section{INTRODUCTION}

The high pressure equation-of state (EOS) of a material can be determined by subjecting it to a steady, fully developed shock wave and making measurements of shock speed and mass elocity fint $^{\text {, }}$ Applying the Hugoniot jump conditions [1] that describe conservation of mass, momentum, and energy across the shock front within the material allows determination of the material's principal Hugoniot curve on its EOS surface. Shocks of this type are typically generated with plate impacts using smooth bore launchers. Impactor velocities of approximately $7 \mathrm{~km} / \mathrm{s}$ with conventional two-stage launcher technology, flyer plate velocities of $10-12 \mathrm{~km} / \mathrm{s}$ (in configurations useful for EOS) on the more advanced Sandia HVL [2], and several micron thick plates to comparable velocities using laser drives [3], however, have historically limited the accuracy and/or pressure states which can be accessed with impact techniques in the laboratory.

Launching a flyer plate in the HVL configuration developed at Sandia is achieved by using a graded density impactor. This technique produces an initial shock loading followed by a time dependent ramp wave, or quasi-isentropic, loading of the flyer plate which attempts to keep the plate temperature at a minimum ( typically $\sim 500^{\circ} \mathrm{C}$ ) and allows it to remain intact during launch. Maximum flyer plate velocities for EOS research are limited by both graded density impactor speeds of approximately $7.3 \mathrm{~km} / \mathrm{s}$ and the inability to propagate a truly shockless, smoothly increasing loading profile into the flyer plate upon impact.

A new capability for producing smoothly increasing pressure profiles using fast pulsed power as the energy source is currently being developed on the Sandia National Laboratories $Z$ Accelerator [4]. The $Z$ Accelerator is a low inductance pulsed power generator capable of 


\section{DISCLAIMER}

This report was prepared as an account of work sponsored by an agency of the United States Government. Neither the United States Government nor any agency thereof, nor any of their employees, make any warranty, express or implied, or assumes any legal liability or responsibility for the accuracy, completeness, or usefulness of any information, apparatus, product, or process disclosed, or represents that its use would not infringe privately owned rights. Reference herein to any specific commercial product, process, or service by trade name, trademark, manufacturer, or otherwise does not necessarily constitute or imply its endorsement, recommendation, or favoring by the United States Government or any agency thereof. The views and opinions of authors expressed herein do not necessarily state or reflect those of the United States Government or any agency thereof. 


\section{DISCLAIMER}

Portions of this document may be illegible in electronic image products. Images are produced from the best available original document. 
capacitively storing $11.6 \mathrm{MJ}$ of electrical energy which, when discharged creates currents of 22 MA. When the machine fires, the current is delivered to the central target by 36 transmission lines arranged like spokes on a wheel and insulated by water. The resulting electrical pulse typically achieves powers as high as 24 terawatts into the evacuated center section which contains the load in a configuration useful for launching flyers.

A technique has recently been developed on this accelerator which makes use of these high currents and their resulting magnetic fields to produce 'nearly isentropic loading on $15 \mathrm{~mm}$ diameter samples that are up to $1 \mathrm{~mm}$ thick over approximately $150 \mathrm{~ns}$ [5]. This smoothly increasing pressure source allows a new method to launch flyer plates to high velocities with masses and dimensions comparable to those launched with the HVL. The technique is similar to conventional electromagnetic launcher (railgun) technology [6] except the $Z$ method uses much higher currents, the loading is applied in approximately $200 \mathrm{~ns}$ instead of milliseconds, and the flyer attains maximum velocity in millimeters instead of meters.

In addition to the high velocities and impact quality of the launched plate that can be achieved using this technique, 4 to 8 plates can be launched simultaneously in a single firing of the $Z$ accelerator. The technique has been demonstrated for aluminum, copper, and titanium flyer plates, and attempted with sapphire flyers. This new capability has application to many problems of interest within the hypervelocity launch community. The primary interests are equation of state measurements, debris shield development for spacecraft, and hypervelocity lethality. With present and projected impact velocities, impact quality, and relevant sample dimensions possible on $\mathrm{Z}$, new pressure regimes for material response can be accessed with gas gun accuracy (including applications to liquid $\mathrm{D}_{2}$ [7]). In addition, hypervelocity impact lethality can be investigated on up to four targets having different geometries simultaneously with very similar impact velocities and impact quality since each flyer plate experiences essentially identical loading.

\section{EXPERMMENTAL TECHNIQUE}

The method by which a magnetic field is used to launch flyer plates on the $\mathrm{Z}$ accelerator is shown in Figure 1.

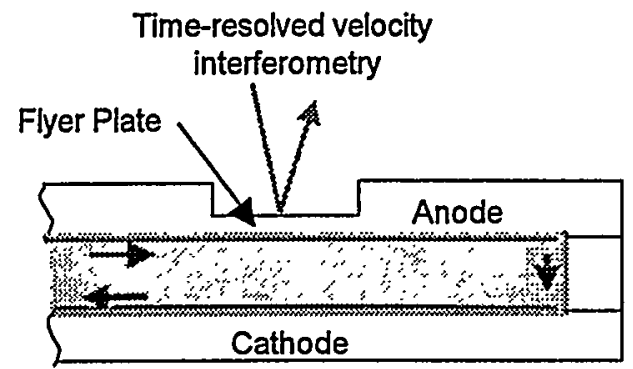

(a)

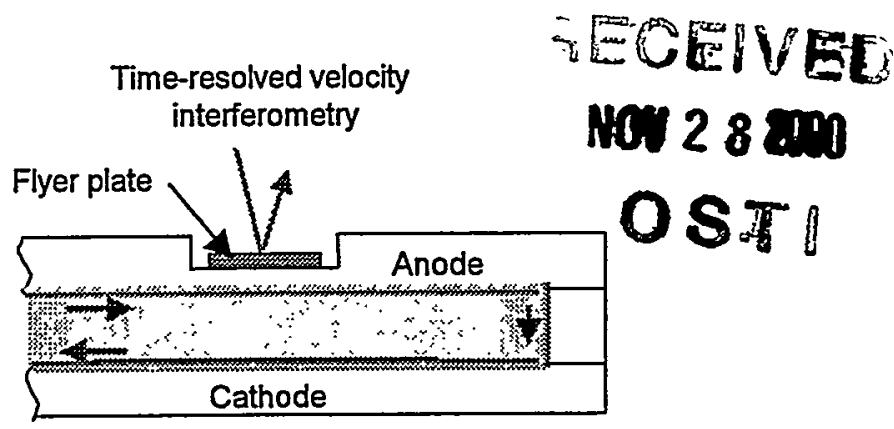

(b)

Fig. 1: Illustration of how magnetic field formed in the vacuum insulation gap between the anode and cathode on the $\mathrm{Z}$ accelerator is used to launch flyer plates. Fig. 1a shows the configuration used for the aluminum plate launch (Z574) and Fig. 1b shows the configuration used for both titanium (Z592) and copper (Z574).

A short circuit is created between the anode and cathode in the $\mathrm{Z}$ accelerator that results in a current flow on their respective inner surfaces. The interaction between the current density and magnetic field produced in the insulating gap due to the current flow produces a time dependant pressure that is applied to the inner surface of the flyer plate. The magnitude of this loading is given by 


$$
P(t)=B^{2} / 2 \mu_{0}=\mu_{0} J(t)^{2} / 2
$$

Where $P(t)$ is the time dependant magnetic pressure applied to the sample, $J(t)$ is time dependant current density (amps/unit length) at the sample location, $B$ is the magnetic field strength, and $\mu_{0}$ is the magnetic permeability of free space. As can be seen, the pressure will follow the risetime of the applied current profile while its magnitude is altered by modifying the current density. A typical current profile used to launch flyer plates on the $\mathrm{Z}$ accelerator is shown in Figure 2.

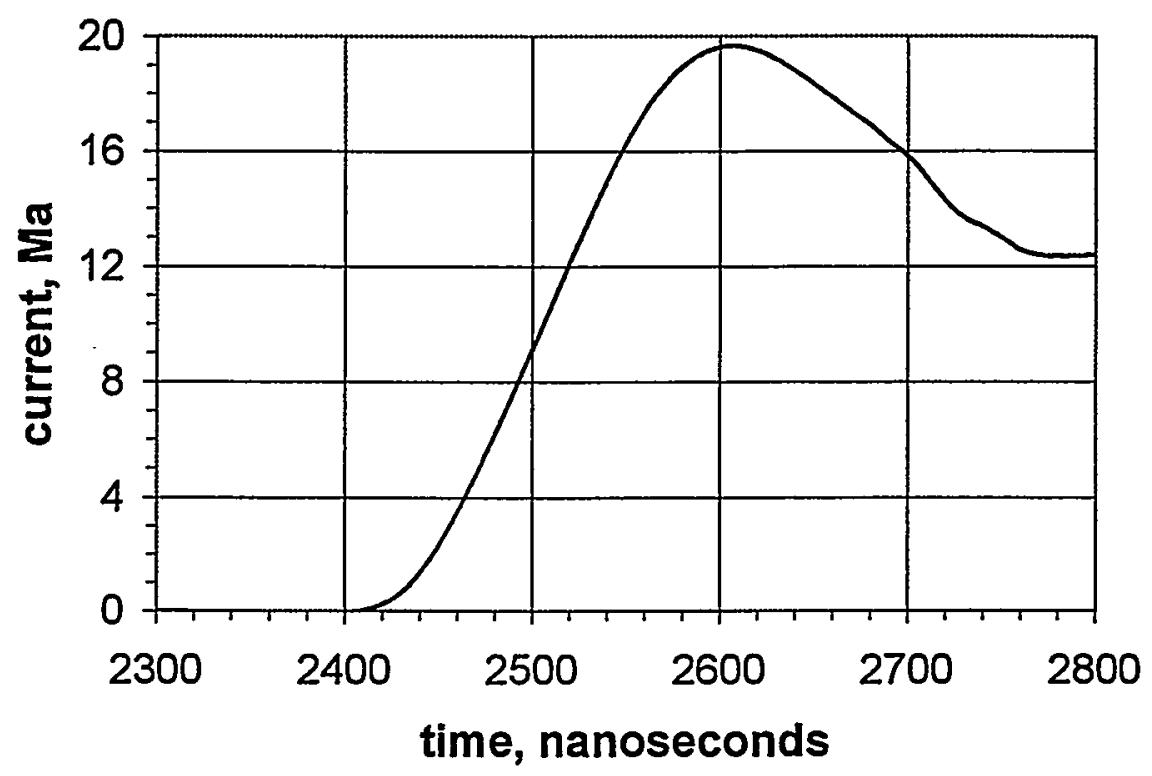

Fig. 2. A typical current profile used to launch flyers on the $Z$ accelerator.

The final velocity of the flyer can be estimated analytically, without the effects of material ablation, through a form of the impulse-momentum equation where $\mathrm{P}$ is the magnetic pressure accelerating the plate per unit area, $m$ is the mass of the plate, $V_{0}$ and $V_{F}$ are the initial (zero for this application) and final flyer velocities respectively.

$$
\frac{\int_{t_{0}}^{t_{f}} P d t}{m}-V_{0}=V_{f}
$$

This relationship provides only an estimate of the final velocity. Computer simulations which include all the experimental parameters and physics are required for more accurate velocity predictions. For a given experimental geometry and a time resolved current profile measurement using Bdot probes [8] from the $Z$ accelerator, equation 1 predicts a time resolved pressure pulse of about $150 \mathrm{~ns}$ duration. It follows that the final velocity of the flyer can be increased by increasing the impulse imparted to the plate or by decreasing the mass. In addition to the impulsive loading on the plate due to the magnetic pressure, material ablation on the currentcarrying surface of the flyer provides an additional impulse. As the current begins to decrease after peaking, the magnetic pressure decreases (from equation 1) and the plasma formed by current diffusion is released from the rear of the flyer and allowed to expand. Conservation of momentum is then evoked causing momentum imparted to the flyer as shown in equation 3 with 
$\mathrm{m}_{1}$ and $\left|\mathrm{V}_{1}\right|$ being the mass and velocity of the ablated plasma (which is time dependant), and $\mathrm{m}_{2}$, $\mathrm{V}_{2}$ are the mass and velocity of the flyer.

$$
\frac{\int_{t_{0}}^{t_{f}} m_{1}\left|V_{1}\right|}{m_{2}}=V_{2}
$$

Simulations performed using ALEGRA, a Sandia National Laboratories MHD code under development, indicate that ablation increases the final velocity of the flyer by approximately $15 \%$ using the typical current profile shown in Figure 2. Since the entire launch process is quite complex, MFD simulations are helping in the understanding of processes involved in the launch cycle.

For applications to EOS studies, a set of four panels is constructed for each experiment and arranged as shown in Figure 3.

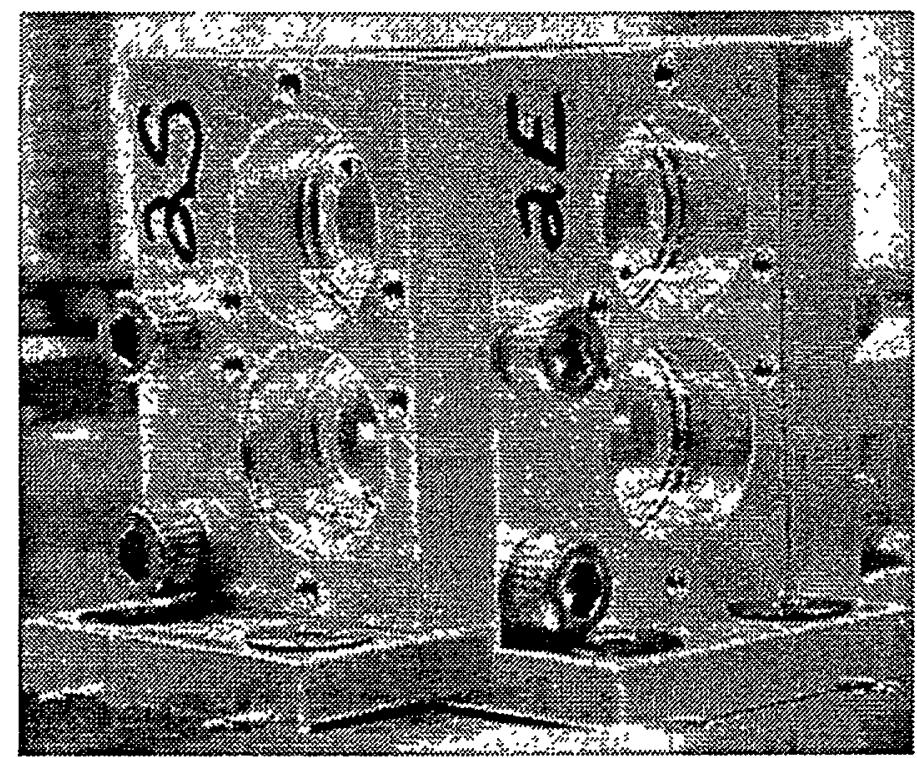

Fig. 3. Picture showing the panel arrangement used to launch flyers on $Z$. Eight flyers can be launched simultaneously because each of the four panels contains two separate flyers.

Flyer plates are created by machining two $10 \mathrm{~mm}$ diameter counterbores into the panels with prescribed material thicknesses of $400-725$ microns remaining as shown in Figure 1a. When exposed to the magnetic pressure confined in the vacuum gap, this thick foil is launched, thereby becoming a flyer. An alternate configuration employs a thinner foil that acts as a drive plate to launch a separate, attached flyer as shown schematically in Figure 1b. The current carrying surface of each panel and the bottom of each counterbore are flat to $200 \mathrm{~nm}$ and parallel to $2 \mu \mathrm{m}$ with $20 \mathrm{~nm}$ surface finishes. Each of the four panels contains two separate flyer plates allowing eight to be launched simultaneously during a single firing of $Z$. The panels are assembled onto the anode plate forming a symmetric gap about the square cathode post.

It is critical that the gap between the panels and cathode post be uniform around the periphery. Uneven spacing between the anode and cathode panels produce local inductance variations which lead to increased current flow (increased $J$ in equation 1 ) in areas with thinner gap spacing, and therefore, uneven pressures across the surface of the flyer. In general, the more evenly pressure is applied to the flyer plate, the higher the probability of a successful launch. Simulations of the magnetic pressure uniformity as a function of flyer plate radius in this panel configuration were 
performed using the EM code QUICKSILVER [9]. The results, shown in Figure 4, indicate that pressure gradients of $\sim 4 \%$ could be expected across the center $7 \mathrm{~mm}$ of the flyer diameter and increase to $\sim 20 \%$ with increasing radius. These gradients will cause local velocity variations across the flyer surface leading to plate distortions that may amplify with flight distance. It is not yet clear whether this is a stable or unstable perturbation.
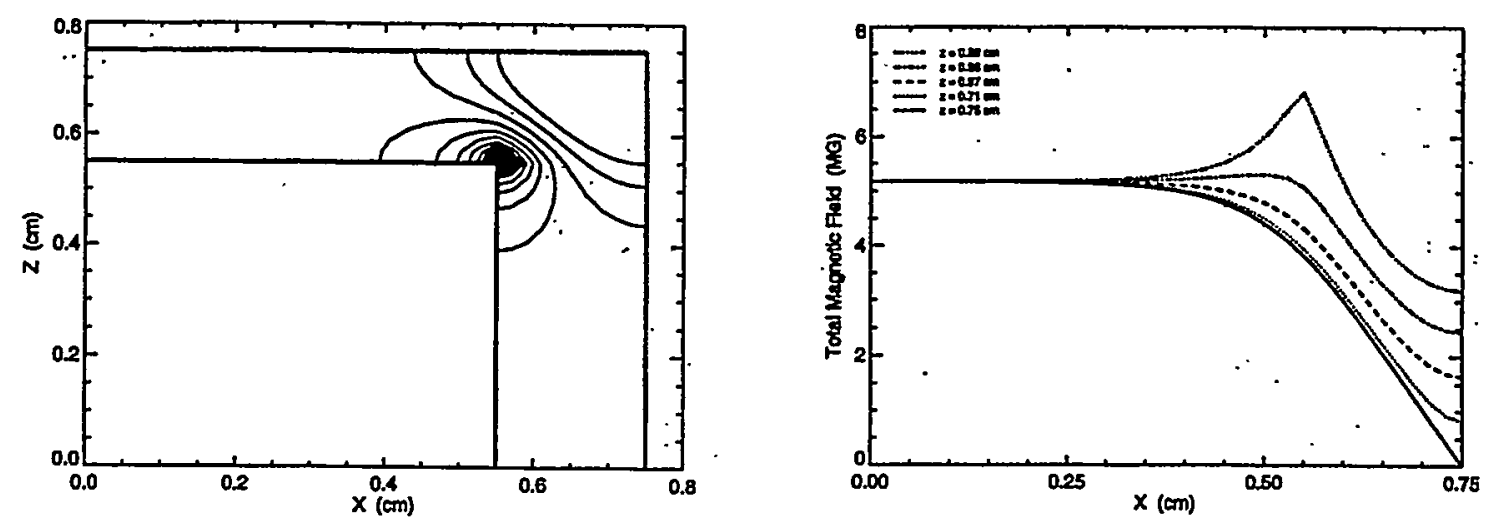

Fig. 4. Simulations of magnetic field uniformity acting on the rear of the flyer plate. The field, which can be converted to pressure using equation 1 , is confined within the evacuated anodecathode on $Z$.

The uniformity of the magnetic pressure acting on the flyer appears to be time dependent as well. As the compression wave reaches the front of the flyer and imparts velocity to this surface, it begins to accelerate. Since the flyer and panel are machined from one piece of aluminum with no parting line currently provided, the edge of the flyer cannot move at the free surface velocity until it has broken free from the panel. During this process, the center of the flyer has already moved forward unimpeded causing plate deformation. This deformation causes asymmetries in the anode-cathode insulating gap, which translates into uneven pressure distributions across the panel face until the entire flyer is free to move. Independent of normal edge effects, this process gives rise to velocity gradients within the flyer which result in curvature of the plate.

The flatness of the launched flyer plate is also strongly influenced by edge waves moving at the launched material's. sound speed for the entire flight time. This can cause significant plate deformation as the release waves lower the pressure of the material they travel through causing velocity dispersion in these regions. Attempts to overcome this phenomenon have been made by Chhabildas [2] through the use of sacrificial rings launched with the flyer. An alternate approach is to increase the diameter of the flyer until the unperturbed region is sufficiently large to achieve the desired impact conditions in the central impact area.

Simulations have also been performed using ALEGRA to help understand current diffusion in the flyer or drive plate. The material through which current has diffused will form a plasma with elevated temperatures and decreased densities. If current diffuses through the entire flyer plate thickness, it will no longer be a solid with known impact characteristics and, therefore, is not an effective EOS impactor. The 1-D MHD simulations were used to define a final material thickness of 175-225 microns, depending on material, to be launched at essentially ambient conditions without the effects of current diffusion. Three geometries were considered; 1) a thick aluminum plate, 2) a copper flyer plate attached to an aluminum driver, and 3) a titanium flyer on an aluminum driver. Plate thicknesses were chosen to provide impact velocities and final thicknesses (after any material is lost from ablation) capable of creating 2.5 Mbars in a 200 micron thick aluminum target with a 20 ns constant pressure pulse on its rear surface. In all cases, the 
experimental profile shown in Figure 2 was used. Results of the MHD simulations are shown in Figure 5.
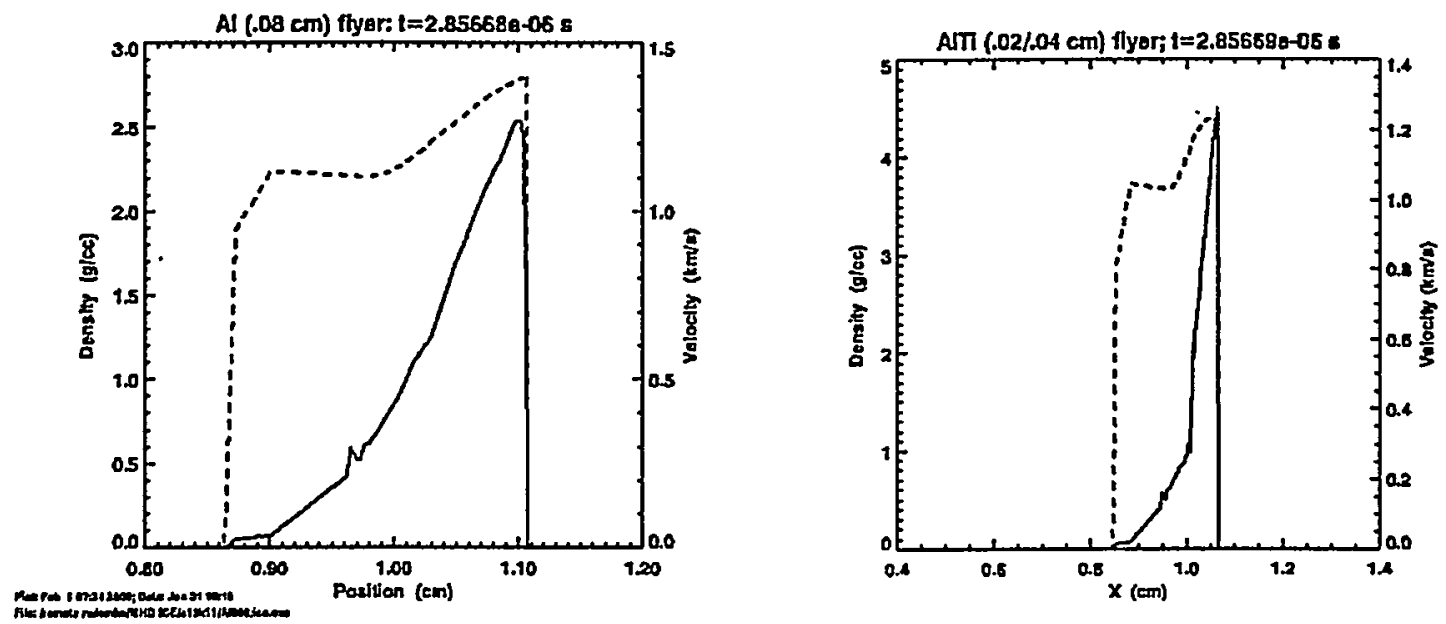

Fig. 5. ALEGRA simulations of flyer plate density profiles after flight distances of approximately $3 \mathrm{~mm}$. The simulations predict less than ambient density for aluminum and a very thin titanium plate upon impact.

In general, results suggest that some current diffuses completely through the flyer plates. In the case of aluminum, Figure 5 indicates that only about $100 \mu \mathrm{m}$ of material remains at a density of $2.5 \mathrm{gms} / \mathrm{cm}^{3}$. This current flowed on an anode perimeter of $6 \mathrm{~cm}$ at the flyer locations creating current densities of $\sim 3 \mathrm{MA} / \mathrm{cm}$ and peak pressures of $\sim 1 \mathrm{Mbar}$. Areas of uncertainty in these calculations include the conductivity model over temperatures ranging from ambient to several $\mathrm{eV}$, which are probably generated on the current carrying surface due to ohmic heating. Values of $3.7 \times 10^{7} \mathrm{mhos} / \mathrm{m}$ were used for conductivity [10]. Also, the EOS of aluminum in this warm, dense matter regime is not well experimentally validated. The model used in these simulations was from the CTH reference manual written by G. Kerley [11]. Each of these parameters appears to have a strong effect on the calculations of current diffusion, so measurements were made in the flyer launch experiments to validate these predictions.

\section{EXPERIMENTAL RESULTS}

Data for each experiment was gathered using three different diagnostics; conventional VISAR [12], a Line Imaging VISAR [13], and fiber coupled shock arrival sensors. In combination, results from these diagnostics can provide information about flyer velocity and integrity. In each experiment, two flyer plates, assumed to be identically launched, were diagnosed and compared. Preliminary data suggests that this is justifiable although VISAR measurements of all flyers on a single shot will ultimately be made to ensure the validity of this assumption.

The Line Imaging VISAR used in these studies images the flyer through a system of lenses onto a slit located at the entrance to a streak camera. This slit was arranged horizontally on all three flyer shots because simulations using ALEGRA indicated that uniformity of magnetic pressure used to launch the flyer was worst along this axis. Results of the streaked data for the aluminum (Z575), sapphire (Z576), and titanium (Z592) flyers are shown in Figures 6, 7, and 8 respectively. Time and spatial scales are indicated on each image. The alternating light and dark lines in the image represent interference fringes superimposed onto the flyer. Changes in fringe position can be related to velocity through the velocity per fringe constant that is preset into the VISAR $(\sim 4.42 \mathrm{~km} / \mathrm{sec} /$ fringe for the present experiments). Thus, the parallel lines at the bottom 
of the streak indicate no motion. As the fringes shift at the position indicated, the flyer has begun to accelerate. In other words, fringe displacement is proportional to velocity.
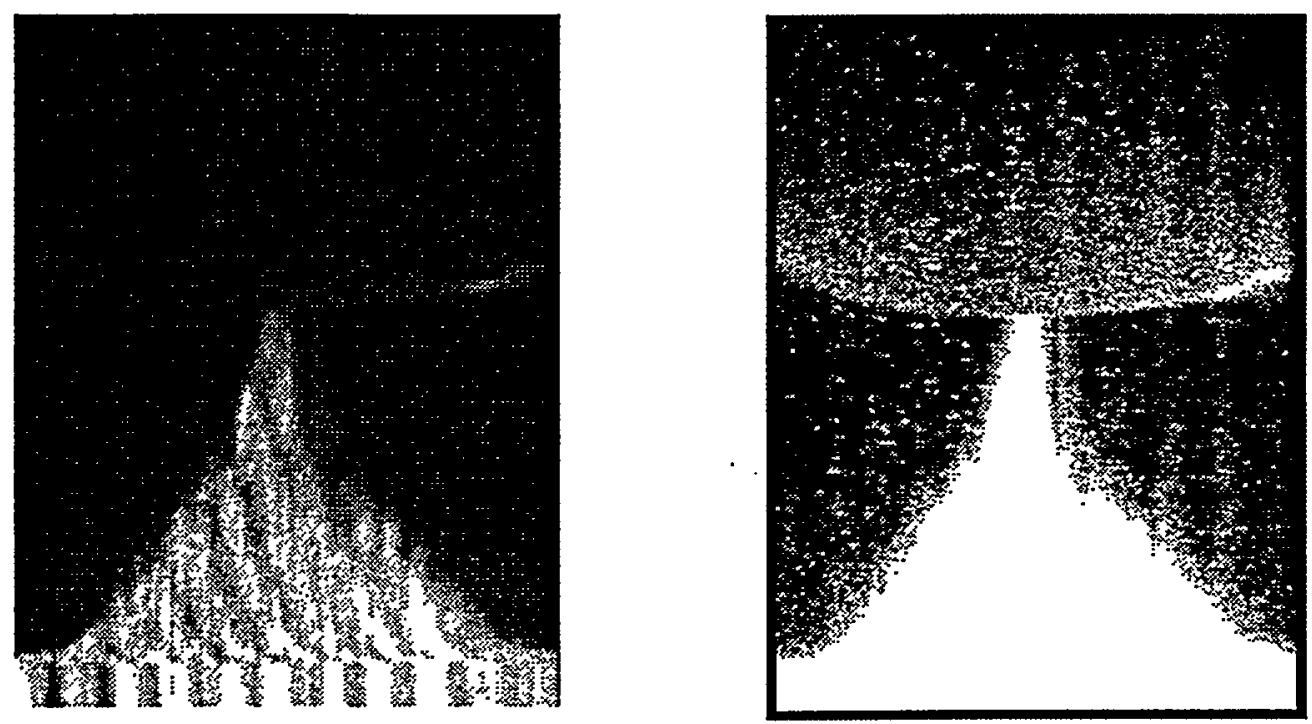

Fig. 6. A streaked Line VISAR image of the aluminum flyer launch. The contrast was increase on $6 \mathrm{~b}$ to more easily see the emission from impact onto the LiF disk.
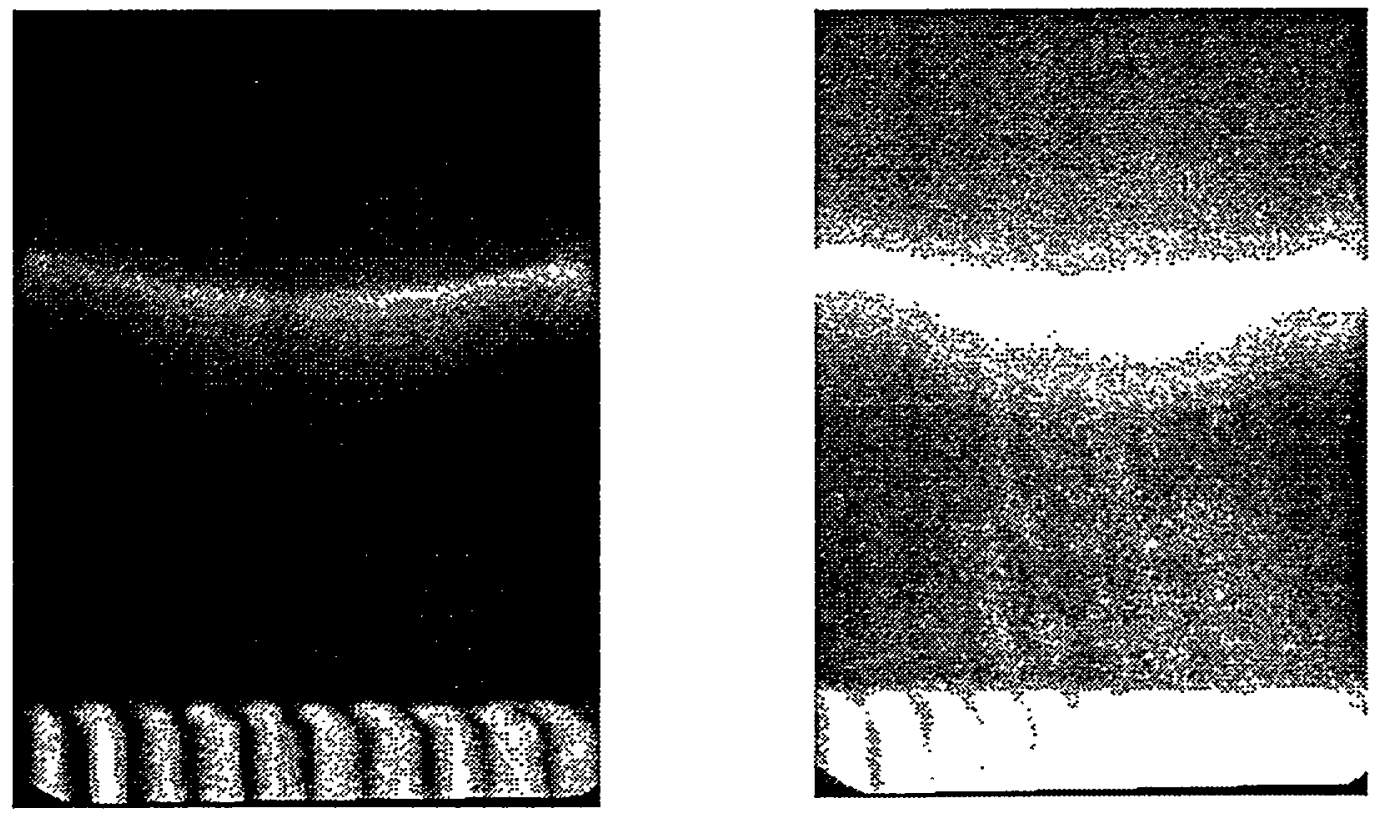

Fig. 7. A streaked Line VISAR image of the attempted sapphire flyer launch. The contrast was increase on $7 \mathrm{~b}$ to more easily see the emission from impact onto the $\mathrm{LiF}$ disk. The flyer failed during the launch process. 


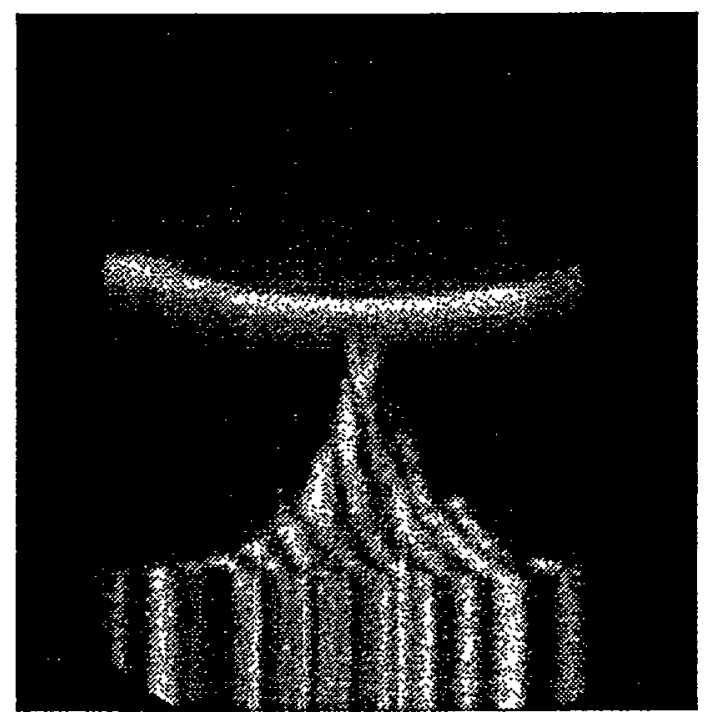

Fig. 8. A streaked Line VISAR image of the titanium flyer launch and impact onto a LiF disk.

As the flyer begins to distort due to edge perturbations, the Line VISAR optics can no longer collect the reflected light from the specular flyer surface because they are relatively slow (f/8) (i.e. the return light is reflected off the collecting lense). This leads to the loss of fringe information that is observed as the flyer propagates toward the target. There is a gradual bowing of the plate that increases with time for the aluminum and titanium flyers, but a catastrophic loss of reflectivity at an earlier time for the sapphire plate. It is thought that the sapphire internally yielded upon pressure release at the free surface causing the flyer to fracture. The data on the two metallic plates strongly suggests, however, that the specular surface is unperturbed during the launch cycle allowing the center region of the plate to remain reflective and provide velocity information over the entire launch cycle. This also provides an indication that the sample is remaining at close to ambient temperatures because, within the resolution of our streak camera, the reflectivity does not appear to change. Theoretical work by Ujihara [14], compared to available data, suggests a change in reflectivity of $20-30 \%$ as aluminum increases in temperature from ambient to melt at $\lambda$ $=690 \mathrm{~nm}$. He also concludes that as $\lambda$ decreases, the percent change in reflectivity for aluminum will increase. With $\lambda=532 \mathrm{~nm}$ in these experiments, I would expect the $20-30 \%$ change in reflectivity to be a lower bound and detectable with our streak camera system. Velocity profiles obtained for aluminum and titanium flyers from the Line VISAR are shown in Figures $9 \mathbf{a}$ and $\mathbf{b}$ with peak velocities for each of the flyer configurations given in Table 1. The copper flyer was monitored using only a conventional VISAR with the resulting velocity profile shown in Figure 9c.

The second feature that can be observed in the streak image is the impact of the flyer on the front of a LiF disk initially $2.3 \mathrm{~mm}$ (Z592) or $3 \mathrm{~mm}$ (Z575) from the flyer. The impact causes an emission of light in the crystal that is recorded on the streak, providing a "reversed" image of the flyer curvature. From the curvature of this spatially resolved feature and the velocity of the plate, an effective tilt can be calculated to determine the flyer's usefulness for EOS studies. Results are summarized in Table 1. It appears that the central region of approximately $3 \mathrm{~mm}$ diameter is flat to within about 4 milliradians in each case, with increased bowing at greater radii.

The impact on LiF that is recorded on the streak camera also provides information about plate integrity. In the case of titanium and aluminum flyers, the impact is distinct indicating a high- 
pressure impact that created a sharp shock. For sapphire (Figure 7), however, the impact appears to be far less distinct and occur over a long period of time indicating a distribution of particles created the shock instead of a solid, intact piece. This is consistent with loss of fringe information due to a loss of reflectivity from the flyer surface early in the sapphire launch.
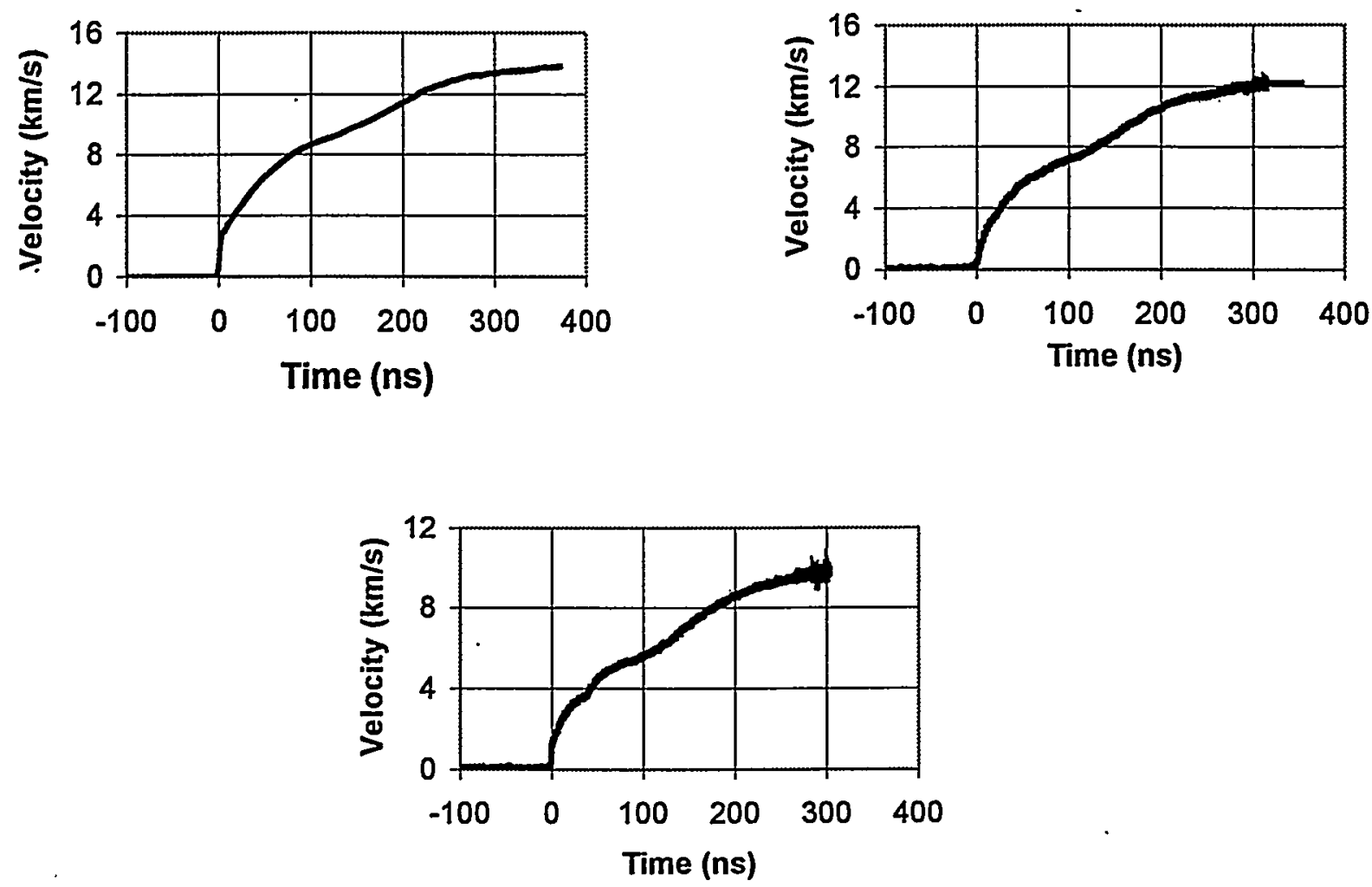

Fig. 9. Velocity profiles for 9a) aluminum with Line Imaging VISAR, 9b) titanium with conventional VISAR, and 9c) copper with conventional VISAR.

Table 1.

\begin{tabular}{|c|c|c|c|c|c|c|c|}
\hline $\begin{array}{l}\text { Shot } \\
\text { No. }\end{array}$ & $\begin{array}{c}\text { Flyer } \\
\text { Material }\end{array}$ & $\begin{array}{c}\text { Drive } \\
\text { plate } \\
\text { (mat'l/thk) } \\
(\mu \mathrm{m})\end{array}$ & $\begin{array}{l}\text { Initial Flyer } \\
\text { Dimensions } \\
\text { (dia. } X \text { thk) } \\
\text { (mm } X \mu \mathrm{m} \text { ) }\end{array}$ & $\begin{array}{c}\text { Flyer } \\
\text { Thickness } \\
\text { at impact } \\
\text { ( } \mathrm{\mu m})\end{array}$ & $\begin{array}{c}\text { Peak } \\
\text { velocity } \\
(\mathrm{km} / \mathrm{s})\end{array}$ & $\begin{array}{c}\text { Effective } \\
\text { tilt } \\
3 m m \text { dia. } \\
\text { (mrad) }\end{array}$ & $\begin{array}{c}\text { Effective } \\
\text { tilt } \\
5 \mathrm{~mm} \text { dia. } \\
\text { (mrad) }\end{array}$ \\
\hline$\overline{\mathrm{Z} 575}$ & alum & $\mathrm{n} / \mathrm{a}$ & 725 & 267 & 13.0 & 4.3 & 38.9 \\
\hline Z575 & copper & alum $/ 500$ & 150 & 181 & 10.0 & N/a & N/a \\
\hline Z592 & titanium & alum/525 & 175 & 217 & 12.3 & 4.1 & 36.8 \\
\hline
\end{tabular}

To evaluate the ALEGRA simulations of current diffusion, a flyer was launched into a $10 \mathrm{~mm}$ diameter aluminum wedge with a $10^{\circ} \pm 0.5^{\circ}$ angle. Fiber optically coupled shock arrival sensors, 
which detect a change in reflectivity of the sample surface upon shock arrival, were placed normal to the surface at $0.7 \mathrm{~mm}$ intervals along the wedge as shown in Figure 10.

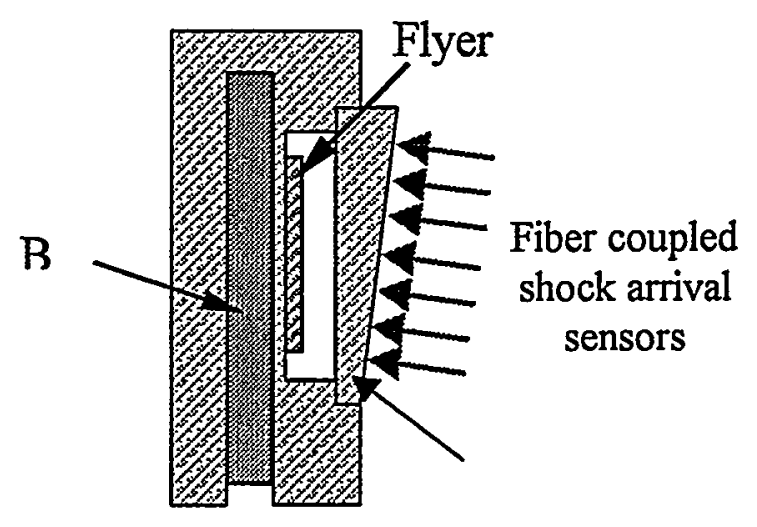

Fig. 10. Illustration of the experimental configuration for impact of the flyer plate onto an aluminum wedge to observe the point where the rarefaction wave overtakes the shock.

The sensor outputs were coupled to a streak camera with a 200 ns window. A typical streak image for a flyer plate impact is shown in Figure 11. The nonsymmetry that is seen in the arrival of the shocks along the wedge is due to the curvature of the flyer upon impact. By using the Line VISAR images showing flyer profiles from impacts on the LiF disks, corrections to the time of impact along the wedge could be made.

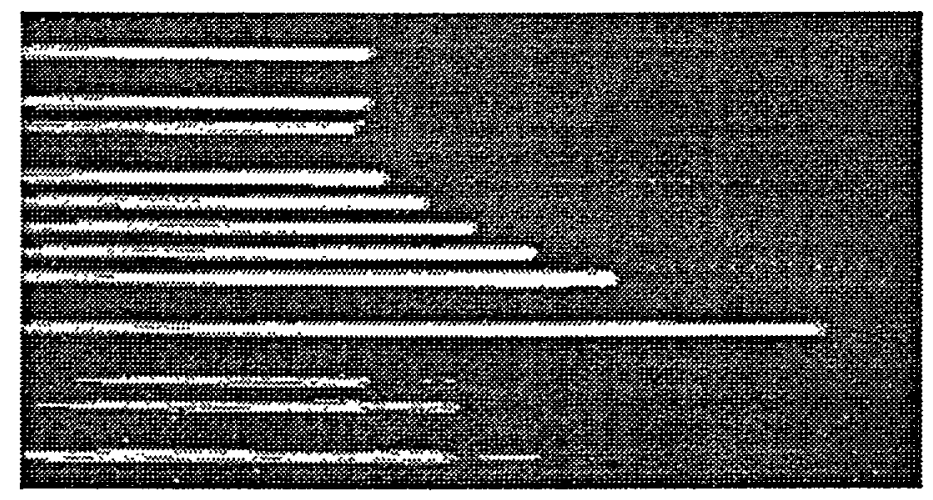

Fig. 11. Typical streak image of fiber optic shock arrival sensor data for arrival of shock along the $10^{\circ}$ aluminum wedge. The shock arrival times must be corrected through estimates of plate curvature.

Shock arrival at the individual sensor positions was plotted for each of the flyers with results shown in Figures 12a, b, and c. The initial linear rise that can be seen in each of the graphs represents the shock velocity in the aluminum wedge for the applied stress state, and indicates constant shock pressure. The point at which the slope change is observed, at an aluminum thickness of $\sim 1.2 \mathrm{~mm}$ in each case, indicates the rarefaction wave from the rear of the flyer has overtaken the shock, resulting in a lower stress state in the wedge. As expected, this new shocked state has a lower wave speed in each of the plots. 

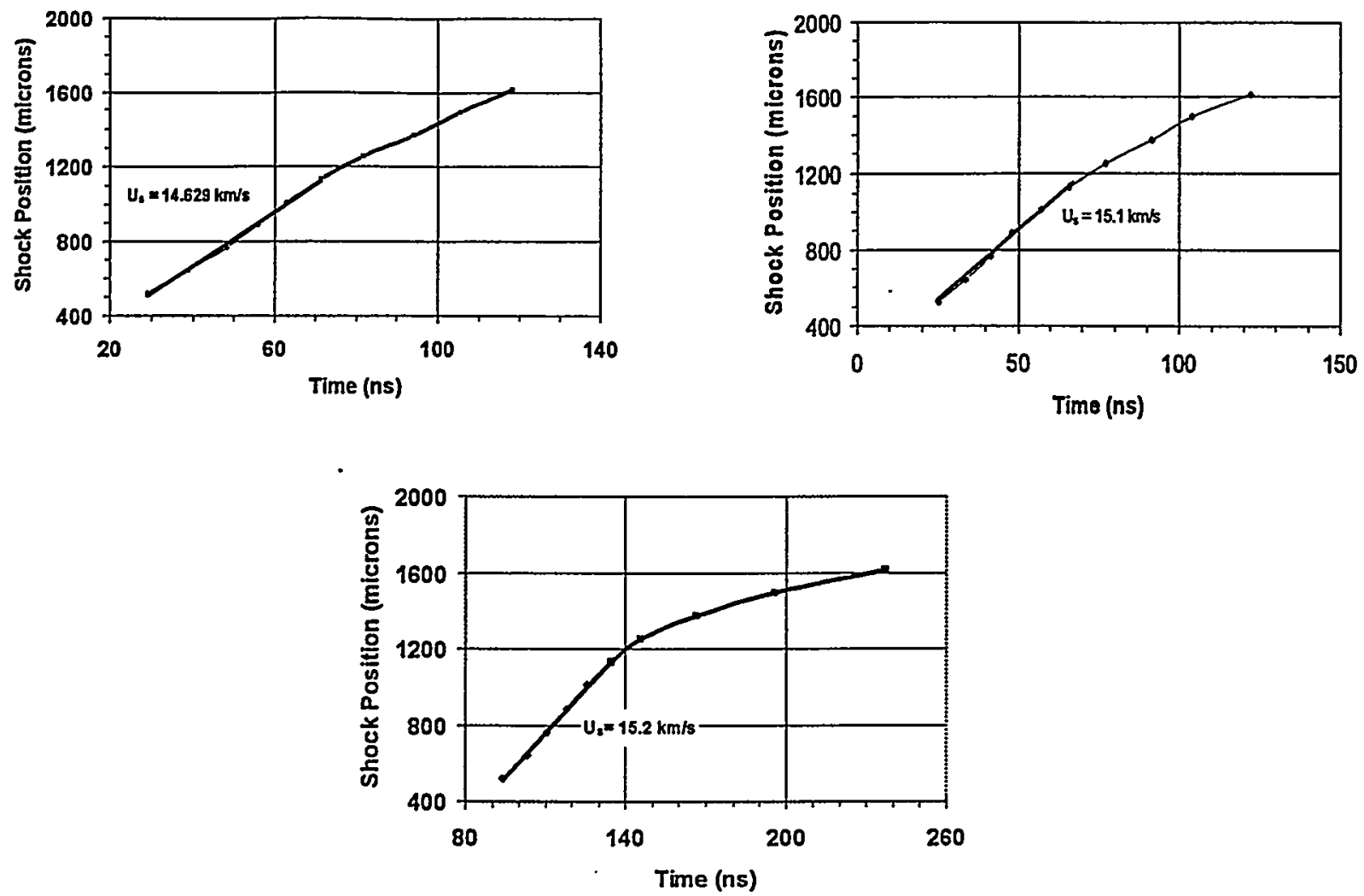

Fig. 12. Shock trajectories inferred from the shock arrival sensor data on the aluminum wedge after correcting for flyer curvature. $13 \mathrm{a}$ is for aluminum, $13 \mathrm{~b}$ for titanium, and $13 \mathrm{c}$ for copper.

Analysis of this inflection point allows determination of the flyer thickness to first order assuming it remains close to an ambient state on the impact surface. This analysis assumes that the speed of the rarefaction wave overtaking the shock can be approximated by

$$
\mathrm{C}_{\mathrm{L}}=\mathrm{C}_{0}+2 * \mathrm{~S}^{*}\left(\mathrm{u}_{\mathrm{p}}\right)
$$

where $C_{L}$ is the Lagrangian sound speed of the wave, $C_{0}$ is the bulk velocity, and $S$ is the slope of the linear $U_{s}-u_{p}$ relationship for the given material. This approximation for $C_{L}$ is obtained by differentiating the Hugoniot $U_{s}-u_{p}$ relationship $\left(U_{s}=C_{0}+S^{*} u_{p}\right)$. The estimation for plate thickness with this approach has several potential sources of error in addition to the rarefaction wave speed ( $\sim 5 \%)$. By making ten discreet point measurements of shock arrival along the wedge instead of a continuous measurement, an inflection point must be interpolated with linear approximations to the two different slopes in the graphs of Figure 12. This can be determined to $\sim 5 \%$ accuracy. Errors in the placement of sensors along the wedge $(\sim 10 \%)$, and the corrections for plate curvature from Line VISAR on LiF impacts (20\%), can also affect this analysis. A RMS error for plate thickness from this data is estimated to be on order of $25 \%$. Values for flyer thickness listed in Table 1 indicate a thicker copper and titanium flyer than was initially launched. This additional thickness is within the defined experimental error. It is interesting to note that ALEGRA simulations using the previously described EOS and conductivity models predicted a flyer with less than ambient density (some current had diffused completely through the flyer) for the aluminum plate, and a very thin region of near ambient density material for the titanium flyer. To within the accuracy of the data, shock velocities obtained from the data indicate ambient densities upon impact and thicker plates than predicted. Extrapolating the linear $U_{s}-u_{p}$ 
relationship for 6061-T6 aluminum reported in the LASL Shock Hugoniot Data compendium [15], with $\mathrm{C}_{0}=5.35$ and $\mathrm{S}=1.34$, shock speeds in aluminum were calculated to be consistently less by $\sim 4-5 \%$ for the reported impact velocities and flyer materials. This indicates the impactor is a near ambient solid, as opposed to the lower density ALEGRA predictions. It is important to note, however, that the simulations have only been performed in 1 and 2-D. It is conceivable that a 3-D effect, rather than effects related to EOS and conductivity models, or the code's handling of ablation, could be responsible for the discrepancies. Work is ongoing to determine the nature of these differences.

\section{CONCLUSIONS}

In conclusion, aluminum, titanium, and copper flyer plates have been successfully launched to high velocities using fast pulsed power on the Sandia National Laboratories $Z$ accelerator. The flyers, which are of adequate size to provide accurate EOS information using currently available instrumentation, are approximately $9 \mathrm{~mm}$ in diameter and several hundreds of microns thick in all cases. The flyers were monitored throughout the entire launch cycle with either conventional or spatially resolved velocity interferometry. Velocities of $\sim 13 \mathrm{~km} / \mathrm{s}$ for aluminum, $\sim 12 \mathrm{~km} / \mathrm{s}$ for titanium, and $\sim 10 \mathrm{~km} / \mathrm{s}$ for copper have been demonstrated. Flyer curvature was characterized using a streaked image of the emission created upon impact with a LiF disk. Effective flyer thickness was also experimentally determined by observing the point at which the rarefaction wave from the rear of the flyer overtook the shock in an aluminum wedge. Based upon minimal changes in surface reflectivity and measured shock velocities in an impacted disk of aluminum, the data suggests that a useable fraction of these flyers is in a solid state at ambient conditions.

As many as eight individual flyers can be launched to these velocities in a single firing of $Z$ allowing multiple samples to be compared with identical shock inputs. Experiments are in progress to increase the magnetic pressure that loads the flyers by increasing the current density on the conductors through changes in experimental configuration. Scaling suggests that velocities much higher than $20 \mathrm{~km} / \mathrm{s}$ are possible on $\mathrm{Z}$ with plates of this size or large

\section{REFERENCES}

[1] M.R. Boslough and J.R. Asay, Basic Principles of Shock Compression, High-Pressure Shock Compression of Solids, ed. by James R. Asay and Mohsen Shahinpoor, Springer-Verlag, New York, (1993)

[2] L.C. Chhabildas, J.E. Dunn, W.D. Reinhart, and J.M. Miller, An Impact Technique to Accelerate Flier Plate Velocities to Over $12 \mathrm{~km} / \mathrm{s}$. Int. J. Impact Engng. 14, 121-132 (1993).

[3] D. L. Paisley, "Laser-driven miniature flyer plates for one-dimensional impacts at $0.5-6 \mathrm{~km} / \mathrm{s}$," Shock-Wave and High-Strain Rate Phenomena in Materials," EXPLOMET, eds. M. C. Meyers, et al., eds, Marcel Dekker Inc. 1992.

[4] K.M. Matzen, Z pinches as Intense X-ray Sources for High-Energy Density Physics Applications, Physics of Plasmas. 4 (5), 1519-1527 (1997)

[5] C.A. Hall, J.R. Asay, et.al., Isentropic Compresson of Solids Using Pulsed Magnetic Loading, submitted to Review of Scientific Instruments for publication, 5/99

[6] R.P. Askew, B.A. Chin, et.al., Rail and Insulator Erosion in Rail Guns, IEEE Transactions on Magnetics, Vol. MAG-22, (6), 1986, pp. 1380-1385

[7] Paper submitted by M.D. Knudson, this conference

[8] W.A. Stygar, R.B. Spielman, et.al., D-dot and B-dot Monitors for Z-Vacuum-Section Power-Flow Measurements, Proceedings of the $11^{\text {th }}$ IEEE International Pulsed Power Conference (1997)

[9] D.B. Seidel, M.K. Kiefer, et.al., The 3-d electromagnetic, particle-in-cell code, QUICKILVER, CP90 Europhysics Conference on Computational Physics, edited by A. Tenner (world Scientific, Amsterdam, 1991), pp. 475-482

[10] Y.T. Lee and R.M. More, An Electron Conductivity Model for Dense Plasmas, Phys. Fluids, 27 (5) 1273, May 1984

[11] G.I. Kerley, CTH Reference Manual: The Equation Of State Package, SAND98-0947, Sandia National Laboratories, Albuquerque, NM 
[12] L.M. Barker and R.E. Hollenbach, Laser Interferometer for Measuring Velocities of any Reflecting Surface, Journal of Applied Physics, 43, No. 11, 4669 (1972)

[13] W.M. Trott, M.D. Knudson, L.C. Chhabildas, J.R. Asay, Measurements of Spatially Resolved Velocity Variations in Shock Compressed Heterogeneous Materials Using a Line-Imaging Velocity Imerferometer; Shock Compression of Condensed Matter-1999, AIP Conference Proceedings 505, edited by M. D. Fumish, L.C. Chhabildas, and R.S. Hixon.

[14] K. Ujihara, Reflectivity of Metals at High Temperatures, J. Appl. Phys, 43 (5), 1972, pp. 2376-2383

[15] S.P. Marsh, editor, LASL Shock Hugoniot Data, University of California Press, (1980) 